\title{
Louise VANDELAC
}

Sociologue, département de sociologie, UQÀM

(1996)

\section{"Technologies de la reproduc- tion : l'irresponsabilité des pou- voirs publics et la nôtre”}

Un document produit en version numérique par Jean-Marie Tremblay, bénévole, professeur de sociologie au Cégep de Chicoutimi

Courriel: jean-marie tremblay@uqac.ca

Site web pédagogique : http://www.uqac.ca/jmt-sociologue/

Dans le cadre de la collection: "Les classiques des sciences sociales"

Site web: http://classiques.uqac.ca/

Une collection développée en collaboration avec la Bibliothèque

Paul-Émile-Boulet de l'Université du Québec à Chicoutimi

Site web: http://bibliotheque.uqac.ca/ 
Cette édition électronique a été réalisée par Jean-Marie Tremblay, bénévole, professeur de sociologie au Cégep de Chicoutimi à partir de :

Louise VANDELAC

[Professeure de sociologie à l'Université du Québec à Montréal].

“Technologies de la reproduction : l'irresponsabilité des pouvoirs publics et la nôtre".

Un article publié dans la revue Sociologie et Sociétés. Vol. 28, no 2, automne 1996, pp. 59-75.

Autorisation accordée par Mme Louise Vandelac le 19 décembre 2003.

\section{vandelac.louise@uqam.ca}

Polices de caractères utilisée :

Pour le texte: Times New Roman, 14 points.

Pour les citations : Times New Roman, 12 points.

Pour les notes de bas de page : Times New Roman, 12 points.

Édition électronique réalisée avec le traitement de textes Microsoft Word 2004 pour Macintosh.

Mise en page sur papier format : LETTRE (US letter), 8.5'’ x 11'’)

Édition numérique réalisée le 3 mars 2007 à Chicoutimi, Ville de Saguenay, province de Québec, Canada.

\section{Fait avec}

Macintosh 


\section{Louise Vandelac (1996)}

\section{“Technologies de la reproduction : l'irresponsabilité des pouvoirs publics et la nôtre”}

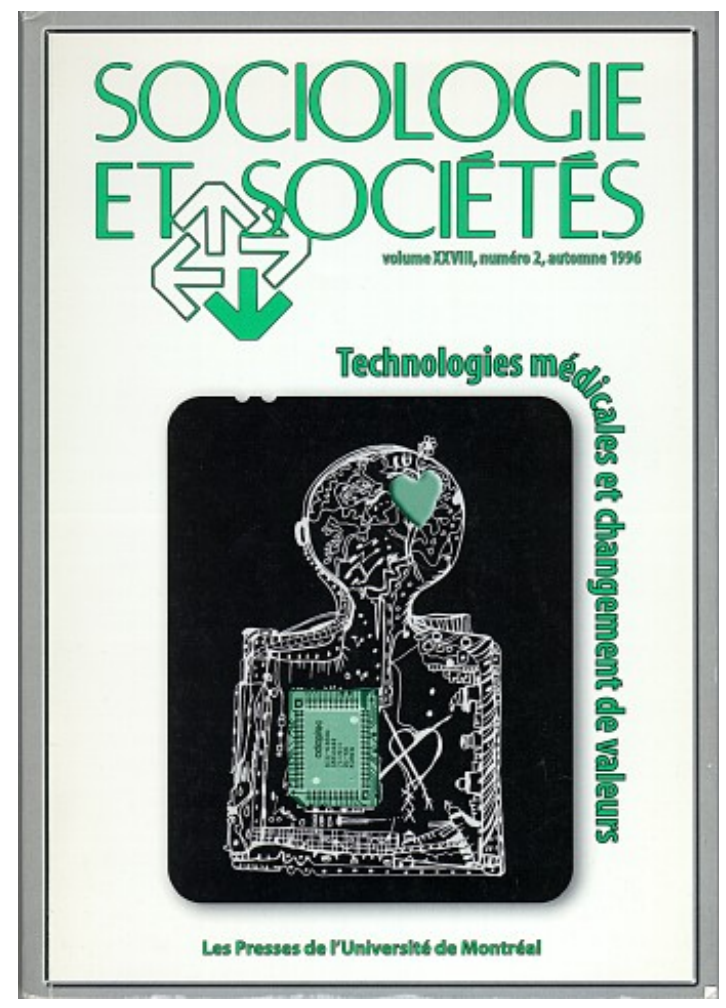

Un article publié dans la revue Sociologie et Sociétés. Vol. 28, no 2, automne 1996, pp. 59-75. 


\section{Table des matières}

Le roman de la technoscience...

Des technologies de reproduction qui font muter l'espèce...

La fin de l'engendrement

Des technologies posant davantage de problèmes qu'elles n'en résolvent...

Penser dans l'évanouissement des repères...

L'absence de politique: la politique du feu vert...

La mystification comme politique...

Résumé

Bibliographie 


\section{Louise Vandelac}

“Technologies de la reproduction :

l'irresponsabilité des pouvoirs publics et la nôtre”. 1

Un article publié dans la revue Sociologie et Sociétés. Vol. 28, no 2, automne 1996, pp. 59-75.

\section{Le roman de la technoscience...}

Le Canada est sûrement l'un des pays dont je me méfie le plus en matière de technologies de reproduction et de génétique : la plupart des scientifiques ont si peu le sens de l'histoire et sont d'une telle naïveté ${ }^{2}$

$\underline{\text { Retour à la table des matières }}$

À la fin des années soixante-dix, le romancier et diplomate Romain Gary publiait Charge d'âmes, fabuleux condensé métaphorique des enjeux de la génétique et du nucléaire, où, sur fond de guerre froide et d'économie technoscientifique, des chercheurs extrayaient l'âme de mourants pour en faire une source d'énergie aussi rentable qu'inépuisable...

1 Je dédie ce texte à J. Hanmer et P. Allen, dont l'article sur les technologies de reproduction publié en février 1979 m'amena initialement à travailler sur ces questions. Je tiens à remercier Abby Lippman, pour sa lucidité, son courage, son sens marqué du partage intellectuel et ses gestes concrets d'encouragement. Bien que ces réflexions ne portent pas directement sur la recherche «Technologies de reproduction, éthique, médias et démocratie », financée par le CRSH, elles s'en inspirent néanmoins.

2 Commentaire d'une généticienne allemande, diplômée de l'université McGill, lors d'une conférence internationale sur les NTR, en Suède, en juin 1985. 
Il y a quatre ans, à Alta Genetics, près de Calgary, haut lieu canadien de production et d'exportation de clones de vaches et de travaux sur l'ectogénèse (gestation artificielle), Willadsen, premier chercheur à avoir créé une chimère chèvre-mouton, me parlait de son projet de roman sur la conservation et la transplantation de cerveaux humains, seul élément du corps, disait-il, qui mérite d'être préservé... À chacun son « âme »...

Quelques mois plus tard, le docteur Barnard, responsable de la première greffe cardiaque, racontait dans une entrevue télévisée son projet de roman sur la conservation de cadavres pour fins de transplantations, véritables " corps sans tête », cette fois, faisant écho aux « têtes sans corps » de Willadsen...

En mars 1994, The New England Journal of Medicine, qui n'a pas l'habitude de faire dans le roman, publiait la lettre d'une médecin intitulée, le plus sérieusement du monde, Cadaveric Ovary Donation (Marcel Mauss doit se retourner dans sa tombe). L'auteure y suggérait d'extraire des ovaires de cadavres pour en faire maturer les ovules, les inséminer et produire ainsi des embryons pour les donner (l'enfer est ainsi pavé de bonnes intentions) aux couples stériles (Seibel, 1994, p. 796). Cette délirante perspective mortifère d'enfants conçus de mères mortes complétait, en quelque sorte, l'image de ces mères mortes, maintenues artificiellement en vie pour assurer la gestation du bébé 3 ; elle prolongeait les projets de Robert Edwards, pionnier de la fécondation in vitro, de transplanter des ovaires d'embryons à des femmes ménopausées (Gauthier, 1990) ; elle renchérissait sur la production d'embryons humains comme matériel de laboratoire à partir d'ovaires d'embryons et de cadavres, comme c'est le cas en Angleterre, où certains évoquent déjà, sous l'euphémisme fetal eggs in assisted fertilisation (Edwards et Benham, 1994, p. 824), l'hallucinante perspective d'enfants conçus d'embryons et donc de « mères » jamais nées...

3 Par souci de concision, je ne peux citer toutes les sources des cas auxquels la presse a largement fait écho depuis une quinzaine d'années. Voir la recherche « Technologies de reproduction, éthique, médias et démocratie », en cours d'achèvement. 
On pourrait ainsi allonger à l'infini la liste des expérimentations scientifiques et sociales qui transforment constamment nos conceptions mêmes du possible, du sensé et du légitime, qu'il s'agisse de demandes de brevets pour du sperme génétiquement modifié ou d'essais de génogénèse... Même le British Medical Journal publiait en décembre 1995 un débat sur l'amélioration génétique de l'espèce humaine, ouvrant ainsi l'épineuse question de la modification des cellules germinales et donc de la transformation du patrimoine génétique de l'humanité, questions qui, encore largement tabou il y a quelques années à peine, donnent lieu, dans certains milieux, à des positions de plus en plus relatives, annonçant déjà des coups de force prévisibles ${ }^{4}$.

Bref, de la fiction romanesque aux fantasmes scientifiques, du roman de la technoscience à l'incarnation de la science-fiction, s'ouvrent, en cette fin de XXe siècle, de bien étranges perspectives. Mais devant cette mise en acte des fantasmes sur les origines et devant cette mise en scène de la production de "l'homme nouveau ", véritables mises en pièces des êtres et de leurs repères identitaires, plusieurs n'y voient qu'imagination débridée, sensationnalisme et enflures verbales Propres à donner froid dans le dos. Bref, tout cela ne serait que du roman.

4 Ainsi, les auteurs-es d'un récent rapport du Comité international de bioéthique de l'UNESCO proposent que les interventions génétiques germinales actuellement indéfendables ne doivent pas pour autant être complètement prohibées "(Germline interventions are indefensible at present, but they should not be categorically disallowed.)»(EDGAR, and TURSZ, 1995), formulation annonçant généralement que d'ici quelques années, les choses seront mûres. 


\section{Des technologies de reproduction ${ }^{5}$ qui font muter l'espèce...}

Retour à la table des matières

Et comme dans ce mouvement emmêlé de réification, d'instrumentalisation et de commercialisation des corps, de leur matière, de leur fonction reproductive, voire de leur descendance, il est de plus en plus difficile de départager le réel et le virtuel et de s'y retrouver dans les dédales techniques, les sirupeux discours de légitimation et les effets de miroirs déformants des acronymes publicitaires et accrocheurs des GIFT, ART, VIP, Life Doctor ou Life Clinic ${ }^{6}$; on s'englue progressivement dans l'illusion du progrès et le miel de "la pensée unique » (Ramonet, 1994), présentés comme autoroute de l'inéluctable.

5 Il s'agit bien de technologies (et non de techniques), qui s'appuient sur un complexe discursif (logos) et qui incorporent, dans l'opacité de leur constitution et les aléas de leur diffusion, non seulement des savoir-faire ou des capacités techniques, mais de la théorie, des intérêts économiques, des choix sociaux, voire politiques, et des principes de différenciation, d'où leur caractère hautement problématique (Limoges, 1988, p. 311). Il s'agit de reproduction et non de procréation, car ces technologies opèrent une rupture radicale avec la procréation (conception sexuée et sexuelle d'un être potentiel singulier) et s'inscrivent dans des stratégies de production sérielle du vivant. Enfin, compte tenu des limites de cet article, nous centrerons notre propos essentiellement sur les technologies d'insémination et de fécondation artificielles et leurs nombreux dérivés technologiques, génétiques et sociaux, et notamment ceux identifiés dans le moratoire volontaire du Canada, tout en excluant l'essentiel du diagnostic prénatal, de l'utilisation des tissus foetaux issus d'avortement, les interventions in utero, etc.

6 GIFT (Gamete Intrafallopian Transfert); fécondation artificielle avec transfert des gamètes dans les trompes, qualifiée de " cadeau »; ART (Artifical Reproductive Technologies); assimilation pour le moins abusive à l'art et à la création ; VIP (Véry Important Pregnancy) ; grossesses assimilées aux personnages très importants qualifiés de VIP (Véry Important People). Quant aux Life Doctor et aux Life Clinic, de Toronto, il est difficile de mieux incarner le mythe prométhéen! 
On laisse ainsi, par ignorance, indifférence, naïveté ou défaitisme, aux prétendus spécialistes ce qui, depuis l'aube des temps, assure la suite du monde et la trame des rapports familiaux et sociaux: l'engendrement, la filiation et leur évolution, alors même que l'enjeu n'est rien de moins que celui de notre propre mutation et celle de l'humanité...

Le biologiste Jacques Testart (1992) et la juriste Marie-Angèle Hermitte (1992) prétendent même que nous aurions déjà commencé à changer d'espèce, expression peut-être excessive pour qualifier l'abolition de certaines frontières de l'espèce humaine, qui annonce déjà une profonde mutation. Ainsi en est-il de cette récente découverte permettant de congeler des cellules souches de la spermatogonie et de les laisser se développer ultérieurement dans une autre espèce, comme des souris, perspective de stockage et de quasi-immortalité particulièrement convoitée pour les champions géniteurs chez les bovins et les chevaux, mais perspective également lourde de sens au plan fantasmatique pour les humains (Kolata, 1996). Chose beaucoup plus banale mais néanmoins fort significative, la fécondation et l'insémination artificielles, déjà responsables de quelques millions de conceptions, ont déjà commencé à altérer profondément la reproduction de l'espèce humaine, faisant passer l'engendrement d'un rapport sexué et sexuel aléatoire avec l'Autre à une reproduction programmée, technicisée, coupée de la sexualité, où l'Autre est souvent réduit à du matériel génétique (sperme ou ovocytes) ou à une fonction instrumentale d'engendrement (mère « porteuse ») ou de gestation (mère gestatrice).

Alors que les enfants étaient issus de la rencontre de deux êtres humains marqués par la différence des sexes, cette "rencontre » peut désormais se réduire à celle d'une personne avec des gamètes, qui, dans le cas de l'insémination avec sperme de donneur (IAD) notamment, a souvent été recueilli à des milliers de kilomètres et à des années de distance pour être traité, analysé, congelé, sélectionné, acheté et ensuite acheminé par la poste... Dans ce processus, généralement marqué de secret et d'anonymat, le " gommage » des " donneurs » ou "vendeurs » de sperme ou d'ovules est délibérément orchestré. En effet, la moitié des traces généalogiques de l'enfant sont effacées, alors que le déni est renforcé par les métaphores médico-administratives transformant les gamètes en " médicaments », l'insémination en " injection » ou le transfert d'embryon en « implantation », encourageant 
d'autant les fantasmes d'autoreproduction narcissique de certaines femmes ainsi que les prétentions des "pères scientifiques " à s'ériger en « nouveaux maîtres de la procréation » 7 .

Il s'agit non seulement de changements biologiques, mais de mutations de sens et de finalité de l'engendrement, de la maternité et de la paternité. Ainsi, malgré la banalisation médiatique dont on l'entoure, donner, vendre ou troquer son potentiel reproducteur implique tout un tissu relationnel et le vecteur de sens qu'est la filiation, ce qui n'est aucunement assimilable à un don de sang ou d'organe. On ne peut donc, par simple volontarisme ou parodie de générosité, parfois contrainte et pervertie ${ }^{8}$, parfois habitée de fantasmes de toutepuissance à peine voilés ${ }^{9}$, réduire l'engendrement à des substrats biologiques et la vie humaine potentielle à du matériel, tout en ignorant la fréquence des troubles psychologiques liés à la filiation et au non-dit, non seulement pour les adultes mêlés à un tel commerce, mais surtout pour leurs descendants, dont les drames psychiques commencent souvent à émerger au moment où ils sont eux-mêmes en âge de procréer (Orenstein, 1996).

Après des générations d'enfants cherchant désespérément la trace de leurs origines biologiques suite à une adoption, voilà que l'État institue et banalise, par le secret et l'anonymat de l'IAD, la possibilité de rendre le géniteur inconnaissable. De surcroît, s'ouvre maintenant le «marché des œufs humains »: une agence d'Angleterre payant des

7 Ainsi, certaines femmes seules recourant à l'IAD disent « se faire un enfant »; d'autres, recourant à la FIV, racontent parfois, «C'est le Dr. Untel qui m'a fait un enfant ! » alors que des directeurs de clinique de fertilité parlent de « leurs 200 ou 300 enfants ", ou déclarent, avec plus de modestie encore, comme le Dr J. Cohen, en France : « En quelque sorte, nous devenons Dieu ! (De Vilaine, 1990, p. 204).

8 Contrainte, par exemple, « lorsqu'on demande à une femme, la veille de son premier transfert d'embryons, si elle accepterait d'offrir ses ovocytes excédentaires à la science. Claude M. a dit oui : «Personne ne m'avait prévenue, je me suis trouvée prise en traître, dans l'impossibilité de dire « non », ce jour même où l'on espère un enfant. Ceci est resté pour moi un point d'horreur. » (De Vilaine, 1990, p. 210 et MFPF, 1989.)

9 Comme ces vendeurs de sperme, pavoisant d'avoir de nombreux rejetons et déjà curieux de voir « leur fille de 18 ans frapper un jour à leur porte »... 
« productrices d'ovules » jusqu'à deux mille dollars (Mc Innies-Rae, 1995); des hôpitaux américains faisant le troc d'ovocytes contre une stérilisation, alors qu'une «clinique de fertilité » de Toronto les échange contre une tentative de fécondation artificielle. Comme le souligne De Vilaine (1990), c'est la première fois, depuis les milliers d'enfants des Lebensborn du régime nazi, nés de pères inconnus et abandonnés par leurs mères dans ces haras humains pour être destinés à l'adoption, et la première fois depuis ces deux cent mille enfants kidnappés dans les pays de l'Est pour être adoptés par des couples nazis, que des États effacent délibérément les traces de la généalogie paternelle de millions d'enfants dans le cas de l'IAD et qu'ils élargissent désormais ce malheur à la généalogie maternelle avec le commerce d'ovocytes, d'enfantement et de gestation, rendant la mère inconnaissable et méconnaissable.

Parler d'abolition des frontières de l'espèce n'est pas non plus un euphémisme quand l'ovulation jusqu'alors modulée par l'entièreté de l'être à travers l'hypophyse peut désormais, sous l'impact de la « castration chimique » provoquée par les agonistes ou anti-agonistes de la LHRH ${ }^{10}$, souvent utilisés en fécondation in vitro, être techniquement contrôlée pour être transformée, fabuleuse expression, en " ovulation pure ", présentée parfois comme une façon de " couper la tête des femmes pour qu'elles ovulent » (Corea, 1993). Cela nous rapproche un peu plus encore du spectre de la médecine vétérinaire, déjà évident dans les pratiques américaines d'insémination artificielle, où tant les " donneurs » animaux qu'humains sont choisis dans des catalogues selon des caractéristiques détaillées et avec photos en prime ${ }^{11}$, ces transferts de l'animal à l'humain étant moins d'ordre technologique que vecteurs de sens, imposant progressivement, à tout l'ordre du vivant, les mêmes schémas économiques implicites...

10 Analogue de l'hormone de libération de la gonadotrophine, agissant comme inhibiteur.

11 Déjà, les banques de sperme américaines offraient des catalogues comprenant la « race » l'origine ethnique, l'âge, le poids, la taille, la couleur et la texture des cheveux, la couleur des yeux, la scolarité, la religion (sic), les « hobbies », le métier, etc. En 1994, Xytex était « fière d'annoncer [qu'elle est] la première et seule banque internationale à offrir en prime la photo des donneurs ainsi qu'un essai personnel où il se décrit » (tiré de leur publicité.) 
Cette " castration chimique », doublée par le forçage de la stimulation ovarienne, fait en sorte que les femmes qui n'avaient qu'un ovule mature par mois en produisent désormais 5, 10 et jusqu'à 40 parfois, devenant ainsi, souligne Testart (1992), les mammifères les plus prolifiques qui soient. Par conséquent, alors qu'elles n'accouchaient, sauf rares exceptions, que d'un seul enfant à la fois, les grossesses multiples, pour les femmes qui deviennent enceintes suite à une FIV, sont maintenant 25 à 30 fois plus nombreuses. Si bien qu'un enfant sur trois, conçu lors d'une FIV, naît d'un accouchement multiple (CRNTR, 1993, p. 595), avec tous les risques et les problèmes de santé qui en résultent pour la mère et l'enfant (Laborie, 1994a et 1994b ; Brunetti et Lancaster, 1989), sans parler des lourds coûts personnels et sociaux qui souvent en découlent.

Au plan individuel, comme au plan collectif, ce sont donc tous nos repères qui s'enfoncent. Ainsi, alors que depuis que le monde est monde, nous avions la certitude d'être nés d'une femme et de mourir, comme le soulignait De Vilaine, voilà que ces deux certitudes s'effritent. Nous pouvons désormais naître d'une femme sans en avoir été tout à fait conçu, la gestation pouvant être enclenchée et menée à partir des ovules d'une autre, introduisant les amères catégories de " mimère ", et peut-être un jour celles de "mère-morte » et de "mèreembryon ». Quant à la mort, voici qu'à la préfiguration des limbes où flottait l'âme des chérubins succèdent les limbes de glace azotées où des êtres potentiels sont suspendus entre une vie et une mort dont le sens même semble dissous par le froid. Quelle troublante impression, confient certaines femmes, qui passent tous les jours, devant l'hôpital où patientent ainsi leurs embryons ${ }^{12}$...

Pour la première fois de l'histoire de notre espèce, des frères et sœurs conçus au même moment peuvent ainsi naître à des années de distance. Ils peuvent également, conçus d'une même mère, être portéses par des mères différentes, comme dans ces contrats de grossesse américains où plusieurs femmes assurent la gestation d'embryons conçus à partir des ovules et du sperme des parents " génétiques » qui

12 Voir L'éprouvante éprouvette, documentaire de Dominique MAGNAN, Paris, 1991, 39 min. G. Productions. 
reprennent les deux, trois ou 4 enfants, portés par les deux ou trois mères différentes qui en accouchent (Basen, 1992).

Bon nombre de ces pratiques dérivent de ce que des médecins se soient autorisés à produire des embryons « en série ", pour pallier leur manque de connaissances et de maîtrise des transferts d'embryons dans l'utérus et pour tenter de réduire ainsi les hauts taux d'échec qui compromettaient la diffusion de la fécondation artificielle (Vandelac, 1989b). Dans une totale perversion de sens, ils ont ensuite parlé d' « embryons surnuméraires »! Souvent transférés à coup de 3, 4, 7 et même 9 parfois ${ }^{13}$, les "surnuméraires » qui auront eu la mauvaise idée de s'implanter en trop grand nombre dans la paroi utérine seront ensuite éliminés par une injection au cœur, provoquant ainsi parfois un avortement de tous les embryons, pratique appelée « réduction embryonnaire » (Berkowitz, 1994). Quant aux embryons non transférés dans l'utérus, ils seront congelés pour transferts ultérieurs et conceptions éventuelles, donnés à un autre couple ou utilisés comme matériel de recherche.

Derrière l'alibi du savoir, nous voilà donc rendus à utiliser d'éventuels rejetons comme nouveaux rats de laboratoire. Cette figure inusitée de l'être potentiel mi-charnel, mi-technique, n'est pas sans rappeler, étrange retour du refoulé, d'autres formes de réductionnisme utilitaristes comme l'esclavage et l'expérimentation sauvage sur les humains, où au nom d'intérêts supérieurs, on institue une nouvelle catégorie d'infra-humains dont l'intérêt économique ou scientifique est qu'ils soient humains, mais qui, s'ils étaient vraiment considérés comme tels, ne pourraient justement pas être instrumentalisés au point de risquer d'être détruits dans l'usage ou l'expérimentation, (Hermine,

13 Malgré l'inefficacité et les risques des transferts de 4 et 5 embryons ou plus observés après 10 ans d'expérimentation sauvage, néanmoins en France, en 1993, 40\% des transferts impliquaient 3 embryons, 17\% en impliquaient encore 4 alors que dans $4 \%$ des cas on a transféré 5 embryons et plus ! Au Mont Sinaï, à New York, sur les 200 premières réductions embryonnaires, 88 étaient pratiquées sur des femmes ayant des triplets, 16 ayant des quadruplés et 7 femmes ayant de 6 à 9 fœtus ! Ces grossesses ont été " réduites » à 189 couples de jumeaux, 5 couples de triplets, 6 enfants uniques, alors que 19 femmes ont perdu complètement tous les embryons (BERKOWITZ, 1994, vol. 3, p. 45). 
1990). Se pose alors cette lancinante question : au nom de quoi ce " destin humain aléatoire » qu'est l'embryon est-il voué, sur l'autel de la technoscience, à ce type de sacrifice ? Et bien que l'espace nous manque pour aborder ici l'épineuse question de l'avortement, soulignons simplement qu'on ne peut mélanger les genres et tout confondre. On ne peut amalgamer les déchirements et la conscience aiguë d'une femme réalisant après la conception qu'elle ne peut donner sens et vie, et qui, comme le souligne Lévinas, ne peut donc porter au sens éthique du terme un nouvel être ; bref on ne peut confondre ce geste, toujours lourd de sens dans la trajectoire personnelle, avec la création et la destruction délibérée de membres potentiels de générations futures sacrifiés sur l'autel d'hypothétiques résultats positifs de recherches, et cela, au nom de ces mêmes générations. Surtout au moment où s'annonce la possibilité de manipuler le génome des embryons, pour les juger, les jauger ${ }^{14}$, en corriger les défauts et éventuellement intervenir dans leurs modalités d'élaboration ; bref, au moment où, rappelle Hermitte, « la domestication de l'espèce humaine est à notre portée ». Cela interroge en effet radicalement notre conception de l'humanité, ainsi que la collusion entre les pouvoirs politiques, scientificomédicaux et médiatiques faisant du " progrès scientifique » une instance au-dessus de tout soupçon, et même au-dessus des lois (De Vilaine, 1990, p. 205).

Tout en se gargarisant de rhétorique « éthique », le Rapport Baird évacue ces questions quand il propose de produire délibérément des embryons pour la recherche (CRNTR, 1993, p. 722), perspective scientiste oublieuse des enjeux psychiques et sociaux, débouchant logiquement sur la production de lignées cellulaires et autres matériaux pour l'industrie pharmaceutique, voire, selon toutes hypothèses, sur la production éventuelle d'embryons génétiquement modifiés, voire brevetés, comme le sont depuis des années déjà, les oncomice.

14 Déjà le diagnostic préimplantatoire permet d'écarter, dès les premières cellules, les embryons non conformes et de transférer le ou les « meilleurs », ultime point de convergence de l'eugénisme négatif et positif, permettant de sélectionner comme jamais notre propre descendance (TESTART, 1992), d'ici à ce que nous puissions génétiquement l'améliorer, comme le propose déjà John A. ROBERTSON (1994). 
Bref, est-il indispensable d'allonger encore la liste pour mettre en évidence la profondeur des mutations anthropologiques à l'œuvre et pour nous demander quels enjeux emmêlés et quelles forces obscures, derrière le paravent du soulagement de l'infertilité (Vandelac, 1988 et 1989a, Gavarini 1987 ; Athéa, 1990) et la myopie d'un certain savoir, poussent ainsi certains pans de la technoscience à modifier nos façons de nous concevoir dans la chair et dans la pensée, au point de transformer d'éventuels descendants en laboratoires vivants ? D'ailleurs, pour paraphraser Vacquin (1990a, 1990b), cette obsession de maîtrise et cette volonté sans limite de déchiffrer, dans le réel des corps, le secret des origines et de l'identité, comme si l'être se repliait terme à terme sur le biologique et que le fameux " connais-toi toi-même » pouvait s'accomplir sur la table de dissection ou dans l'antre de l'éprouvette, comme si une telle réponse pouvait se trouver ailleurs que dans la vie et par l'Autre, ne nous poussent-elles pas à notre propre dévoration?

\section{La fin de l'engendrement...}

$\underline{\text { Retour à la table des matières }}$

Non seulement les frontières de l'espèce se fissurent-elles, mais, obnubilés par l'aveuglante illusion de maîtrise qu'offrent les palliatifs que sont l'insémination et la fécondation artificielles et leurs dérivés, nous ignorons de façon éhontée les sourdes menaces qui risquent de compromettre, d'ici deux à trois générations à peine, la possibilité même de se reproduire autrement que par artifices techniques.

Dès 1982, un article de Mother Jones faisait état des recherches sur la baisse marquée de la qualité et surtout de la concentration des spermatozoïdes, en raison des effets de certains polluants (Castleman, 1982). En 1995, Pierre Jouannet, spécialiste français de la spermatogenèse, publiait une étude rétrospective sur 20 ans menée dans la perspective avouée d'infirmer ces résultats. Menée auprès de 1351 donneurs fertiles d'une banque de sperme de la région parisienne, cette 
étude conclut pourtant, de façon plus dramatique encore, que la concentration de spermatozoïdes a chuté d'environ $2 \%$ par année, faisant passer le taux de 89 millions par millilitre en 1969, à 60 millions en 1992, alors que la qualité (mobilité, normalité) a également baissé (Wright, 1995 ; Auger, 1995 ; Jegou, 1995). En outre, « alors que la concentration du sperme d'un homme parisien né en 1945 était de cent deux millions par m., celle d'un homme né en 1962 était exactement la moitié. [...]. Or, si ce déclin se poursuit au rythme actuel, cela prendra 70 à 80 ans pour atteindre 0 », conclut gravement Jouannet (Wright, 1995, p. 45). En fait, si cette baisse se poursuit au rythme des années 1969-1992, il ne faudra que 32 ans pour franchir le seuil des 20 millions de spermatozoïdes par ml., seuil en deçà duquel, selon l'Organisation mondiale de la santé, la fertilité masculine, encore possible, n'est cependant plus assurée ${ }^{15}$. Ces données ne sont pas propres à la France. En 1992, une recherche danoise portant sur l'examen rétrospectif de 61 études menées dans le monde depuis cinquante ans mettait en évidence une baisse de la qualité du sperme et une chute de 42\% du nombre de spermatozoïdes depuis 1940 (ibid).

Bien qu'on ignore encore avec précision l'importance et les interrelations des différents facteurs responsables, il appert que les oestrogènes synthétisés (xenoestrogènes) dérivés notamment de très nombreux produits chimiques industriels relâchés dans l'environnement et utilisés couramment dans la vie quotidienne, affectent le système endocrinien en déjouant les récepteurs hormonaux (ibid). Cela réduirait non seulement la spermatogenèse, au point d'augmenter l'incidence d'infertilité masculine, mais contribuerait à l'augmentation de malformations génitales et de cancers des testicules, sans parler des fortes présomptions concernant l'impact de ces oestrogènes sur l'endométriose, les cancers du sein et des ovaires (Castleman, 1996; Wright, 1996).

Certes, nous ne saurons qu'à postériori si se poursuivent ou s'accélèrent le déclin de la spermatogenèse et les risques de cancers et d'endométriose. Mais deux ou trois générations sont bien vite passées quand les sources suspectées du problème, inscrites au cœur du développement industriel, sont aussi largement diffusées dans l'environ-

15 Selon Jouannet, l'expérience de l'IAD permet de constater que 5 millions de spermatozoïdes peuvent suffire, alors qu'un un seul est nécessaire avec l'ICSI 
nement et à ce point intégrées dans la vie quotidienne. Comme le souligne Castleman, nous sommes victimes du bain d'oestrogènes dans lequel nous vivons.

Or, comme le fardeau de la preuve incombe, produit par produit, aux scientifiques critiques, alors que l'analyse des effets combinés impliquant des dizaines de produits demeure extrêmement difficile et que l'essentiel des ressources humaines, financières et techniques dans ce domaine sont davantage accaparées par les technologies de reproduction que par les recherches fondamentales en matière de prévention et de protection de la fertilité, il est fort à craindre qu'à moins d'une prise de conscience aiguë des pouvoirs publics, tant nationaux qu'internationaux, doublée d'une rapide intervention et de mises de fonds substantiels, nous soyons piégés par l'irréversible...

Cela pourrait signifier à la fois une crise majeure de la fertilité, la création d'un éventuel marché pharmaceutique centré sur les récepteurs hormonaux ainsi qu'une paradoxale accélération de la technicisation de la reproduction, transformée plus que jamais en véritable marché ${ }^{16}$, entraînant des coûts humains et sociaux exorbitants et des conséquences démographiques, politiques et culturelles qu'on ose à peine imaginer...

Cette accélération technologique en matière de reproduction est évoquée depuis plusieurs années déjà par nombre de praticiens présentant la dégradation de l'environnement comme ultime justification de leurs travaux. D'autres poussent le cynisme jusqu'à dire ouvertement que les causes et la prévalence des problèmes de fertilité importent peu, puisqu'ils ont trouvé des solutions techniques, comme l'ont confié des chercheurs ayant mis au point l'insémination d'un seul spermatozoïde au cœur du cytoplasme de l'ovocyte (ICSI) à la cinéaste Gwynne Basen, lors du Congrès des sociétés américaines et canadiennes de fertilité et d'andrologie tenu à Montréal en 1993. Était évidemment occulté le fait que l'ICSI implique que des femmes par-

16 Déjà le Pacific Fertility Center offre dans le New York Times du 3 mars 1996 un « forfait IVF », qui permet aux femmes acceptées dans le programme d'être remboursées jusqu'à 90\% en cas d'échec de FIV (leurre complet, car les médicaments, les examens et autres services extérieurs (?) sont exclus). 
faitement fertiles subissent une fécondation in vitro lourde de risques et d'effets secondaires, tant pour elles que pour leurs éventuels enfants, sans compter tous les effets potentiels à long terme pour les enfants qui en sont issus (Relier, 1992 ; Laborie, 1992b ; Lancaster, 1991 ; Auroux, 1995)!

Tant en termes de santé publique que d'éthique, négliger ainsi les mesures les plus élémentaires de protection de la santé et des capacités reproductives tout en encourageant la mise en marché de la reproduction, ce qui entraîne une flambée des coûts de santé et une inféodation croissante de l'engendrement aux impératifs et aux catégories économiques (Vandelac, 1990b), interroge profondément notre sens des responsabilités.

\section{Des technologies posant davantage de problèmes qu'elles n'en résolvent...}

$\underline{\text { Retour à la table des matières }}$

Volontairement en deçà des perspectives de développement les plus folles qu'annoncent déjà certains chercheurs (Seidel, 1991 ; Rostand, 1966 ; Kolata, 1996), cette lecture met simplement en évidence que, nonobstant les conceptions qui lui sont imputables et les connaissances acquises, les technologies de reproduction et leurs dérivés génétiques posent plus de problèmes que ceux qu'elles sont censées résoudre. En outre, en centrant les ressources sur des palliatifs plutôt que sur les causes, elles changent profondément le sens de la médecine, la nature de l'engendrement humain, les rapports d'alliance entre les sexes et les générations, tout en transformant des repères essentiels, comme les notions de mère, de père, de vie, de mort et de temps ; bref, elles accélèrent la fuite en avant et font muter la conception de l'humain et de l'humanité, contribuant ainsi à élargir et à amplifier les problèmes.

Il faut bien comprendre en effet que, malgré leur habillage médical - qui fait encore largement illusion - ces technologies ont déjà bouté 
une partie de la médecine hors d'elle-même. C'est d'abord en transformant la nature du problème à traiter qu'elles ont modifié à la fois le rôle de la médecine et le sens de la conception humaine. En effet, ces technologies de la reproduction ne soignent pas les problèmes de fertilité et ne permettent pas aux gens de recouvrer leur capacité reproductive. Elles contournent, par un artifice technique, dans le cas de la FIV, et par un artifice technico-idéologique, dans le cas de l'IAD (Vandelac 1988b), des problèmes réels ou supposés de fertilité, dans le but explicite de produire un enfant.

Le « problème » à résoudre n'est plus alors celui des causes d'infertilité ni même celui du traitement des pathologies et des dysfonctions manifestes. C'est désormais l'absence de conception qui constitue le "problème médical ». Si bien que la demande n'en est plus une de soins, mais bien d'enfant.

Ainsi, en faisant de l' " absence de conception » le " problème médical » et de la production d'enfants potentiels la réponse médicale, la médecine devient «fabrique d'humains potentiels », voire, depuis leur production en série suite à la stimulation ovarienne, productrice de " vivant ", dont certains sont appelés à naître et d'autres à n'être que matériel de recherche, ce qui change radicalement non seulement la nature et le sens de la médecine, mais ceux de la conception des êtres, dans le double sens du terme (Vandelac, 1994b).

Dans cette fabrique du vivant, l'engendrement acquiert donc un nouveau statut de "service médical » de reproduction, au point que, partiellement ou totalement pris en charge par le système de santé public, l'accès aux technologies de reproduction devient pour certaines un "droit », alors que toute limitation à l' " accès à ce service " (y compris pour absence ou refus de partenaire sexuel) est dénoncée comme un acte de discrimination (Vandelac, 1994e)... 


\section{Penser dans l'évanouissement des repères...}

$\underline{\text { Retour à la table des matières }}$

Dans son désir de maîtrise et de transformation du monde comme de lui-même, l'homo faber fait-il taire l'homo sapiens ? (LabrusseRiou, 1994.)

La réflexion, on le voit, ne peut se réduire à des perspectives individualistes de choix (Rebick, 1993) et de droit, et elle ne peut s'enfermer dans les ornières d'un consumérisme étroit de risques et de coûtsbénéfices, paradoxale invitation à plus de performances (Vandelac, 1990b, 1994a). Certes, face à un pan de la médecine, oublieuse de ses fondements et aveuglé par ses pseudo-succès, la critique épistémologique et l'analyse statistique permettent parfois de mettre en évidence la genèse et les enjeux de ces technologies et de s'objecter aux arguments les plus fallacieux, comme ceux des taux de succès (Marcus Steiff, 1994).

Cependant, force est de constater que peu importent la rationalité, l'exactitude ou la clairvoyance de l'argumentation, tout s'éclipse au moindre sourire d'enfant, présenté comme ultime preuve de normalité, d'amour et d'humanisme médical... Au-delà des individus qui recourent à ces technologies, en tentant avec plus ou moins d'information, de réflexion et de bonheur de se déprendre d'une situation souvent ressentie comme envahissante d'impuissance et de désespoir ${ }^{17}$, se pose en effet avec acuité l'incapacité collective de saisir la perversion de sens de cette mutation de l'engendrement en reproduction en série de vivant, voire en " service médical »; l'absence de responsabilité intellectuelle et sociale à l'égard des générations futures et l'incapacité ma-

17 Sans écarter les individus littéralement traversés par ces technologies ni les réduire à des rouages d'une vaste machination, l'espace manque ici pour évoquer ce clair-obscur de l'inconscient et cet entre-chair de l'individuel et du collectif permettant de traduire un tant soit peu la complexité vécue de ces questions. 
nifeste des pouvoirs publics à penser ces questions et à adopter des politiques cohérentes.

Dans cette « mélasse » où tous les délires sont banalisés, toute analyse est facilement taxée d'alarmisme, tout effort prospectif est qualifié de fiction et même les descriptions les plus fidèles sont reçues avec indifférence ou suspicion, comme si les mots n'avaient Plus aucune prise. Comme si, comparativement à la mort, tissant le fond culturel de l'humanité, l'engendrement, qui donne chair et sens aux êtres pour les faire naître à eux-mêmes, n'avait pu être pleinement symbolisé et pensé (De Vilaine et coll., 1986 ; De Vilaine, 1990). Propulsé dans l'expérimentation endiablée alors qu'il nageait encore, idéologiquement, dans les limbes du Discours de Nature (Guillaumin, 1978), c'est comme si l'engendrement n'avait pas réussi à émerger comme trame de la pensée (O'Brien, 1987 ; Irigaray 1984, 1987) et source vivante de «l'engendrement du savoir » (Vandelac, 1994f). Si bien que, démunis pour penser et réagir face à cette mutation, nous restons sans voix face à l'instrumentalsation de l'Autre et à la perversion du sens qui est à l'œuvre dans nombre de ces technologies... Surtout quand, profondément émus et chavirés par les confidences affligées des proches qui n'ont pas eu l'enfant désiré, se pose crûment leur volonté de recourir à une mère porteuse ou à la mythique ectogénèse...

Évidemment, on s'étonne parfois de ces « mamies-éprouvettes 18 » (Clément, 1992) ou autres « nouveautés » occupant ponctuellement la scène médiatique. On s'émerveille bêtement devant des quintuplés, purs effets iatrogènes d'expérimentation débridée ! On est parfois sidéré devant les cafouillages intergénérationnels d'une mère qui accouche des enfants de sa fille ou de sa fille qui donne ses ovules à sa mère ou en porte les enfants, à moins de n'y voir, comme certains médias, que pure générosité ! On est aussi hébété devant les conceptions postmortem avec les gamètes de l'autre conjoint et parfois même le ventre de la belle-sœur, à moins de n'y voir, encore là, qu'ultime preuve d'amour au-delà de la tombe... Et on s'interroge parfois sur la mise en marché des ovules, des embryons et de la gestation, surtout quand des

18 Ces femmes post-ménopausées de 50, 60 ans ou plus qui accouchent d'un enfant suite à un don d'ovocyte et à une fécondation in vitro. 
amies ou des étudiantes sont approchées, y compris dans la rue ou dans les salles de cours.

Mais si individuellement on observe et au mieux on s'inquiète, collectivement on ne s'occupe durablement de rien et on reste muets devant l'incohérence et la courte vue des politiques publiques dans le domaine... Comme si la pensée était en rade et la démocratie en panne.

Au point de se demander si nous ne sommes pas en train de confirmer l'hypothèse d'Edgar Morin « d'un néo-obscurantisme généralisé, produit par le mouvement des spécialisations, où le spécialiste lui-même devient ignare de tout ce qui ne concerne pas sa discipline, où le non-spécialiste renonce d'avance à toute possibilité de réfléchir sur le monde, la vie, la société, laissant ce soin aux scientifiques, lesquels n'en ont ni le temps ni les moyens conceptuels » (Morin, 1990, p. 17).

Comme si nous n'arrivions pas à saisir à quel point les différents dispositifs de pouvoir sur cette nouvelle production du corps individuel et social, marquée par la prégnance du déterminisme biologique et par la biologisation et la " généticisation » du discours social (Vignaux, 1977 ; Lippman, 1992) ; marquée par l'effritement de l'Altérité (Baudrillard, 1995) et par l'indifférenciation du vivant, où s'estompent, voire s'abolissent, les frontières humain, animal, plante : bref, comme si nous saisissions encore à peine la densité et l'ampleur de l'emprise inégalée du bio et de son empire, pressentie de façon prémonitoire par Foucault à travers son analyse du biopouvoir (Foucault 1963 ; Dreyfus et Rabinow, 1984).

Il ne s'agit pas ici simplement d'un déplacement des assises du pouvoir qui, de la possession de la terre et de ses ressources, seraient passées au contrôle de la manufacture et de l'industrie puis à l'empire des communications pour englober désormais la vie et sa production, nouvelle " chasse-gardée des transnationales et des investisseurs de capital-risque » (Decornoy, 1992). L' « artificialisation » et la manipulation croissante des corps et de la conception des humains, véritable « mise en chair » de certaines des représentations réductrices et mécanistes constituantes des sciences modernes (Thibon-Cornillot 1992), 
transforment radicalement la notion même de pouvoir. Car ce qui est en jeu dans ces fantasmes de maîtrise et d'emprise sur l'être, ses origines et ses descendants, c'est le descellement de l'être et de son corps (Deleury, 1995), progressivement utilisé comme source inépuisable de matière première, comme incubateur vivant, comme vecteur de vivant et de vies, susceptibles d'être à leur tour instrumentalisées. En fissurant le socle du réel sur lequel s'élaborent les métaphores corporelles, en effritant les repères psychiques essentiels et en bouleversant les structures symboliques de la société, ce sont non seulement les assises et la notion de pouvoir qui changent, mais notre capacité même de penser (Vacquin, 1987; Vandelac, 1994f).

Face à cette "situation paradoxale, où le développement de la connaissance instaure la résignation à l'ignorance et où le développement de la science est, en même temps, celui de l'inconscience » (Morin, 1990, p. 17), les uns détournent le regard, les autres se voilent la conscience, et tous se déchargent de leur responsabilité en invoquant l'existence de comités d'éthique et d'organismes professionnels chargés de veiller au grain, tout en soulignant que les médecins-ne-fontquand-même-pas-n'importe-quoi 19 et que, somme toute, c'est aux gouvernements de s'occuper de ces questions de sciences et de technologies, de santé et de famille...

Or, justement, l'examen attentif du dossier des technologies de reproduction et des développements génétiques qui y sont liés ${ }^{20}$, met clairement en évidence, non seulement les failles, mais trop souvent la faillite des différents dispositifs (évaluatifs, éthiques, professionnels, administratifs, législatifs, etc.) supposés en examiner le bien-fondé, le sens et la portée, pour ensuite les baliser, les encadrer, voire en interdire les pires dérives (Vandelac, 1995). Si bien que les lacunes, les carences, voire les béances en matière de politiques publiques dans ces domaines ont fait du Canada, non seulement l'un des plus laxistes

19 Ce qui est fort heureusement tout à fait vrai, nos critiques ne visant que certaines orientations de la gynécologie-obstétrique et de la biologie de la reproduction.

20 Cette analyse porte essentiellement sur les pratiques de fécondation et d'insémination artificielles et leurs dérives, ainsi que certaines recherches et interventions géniques visées par le moratoire. 
en la matière, mais " probablement le pays le plus libéral qui soit en matière de recherche biomédicale » (Deleury, 1995, p. 233).

\section{L'absence de politique: la politique du feu vert...}

$\underline{\text { Retour à la table des matières }}$

L'exemple le plus éloquent nous en a été donné par le « moratoire volontaire et temporaire » sur certaines recherches et technologies génétiques et reproductives des plus controversées. Imposé dans l'indifférence générale en juillet 1995, par l'ex-ministre de Santé Canada, Diane Marleau, ce moratoire qui constitue toujours pour l'instant la seule politique effective a été redoublé par la création d'un étonnant comité de surveillance ${ }^{21}$, et il sert désormais de point d'appui au projet de Loi de l'actuel ministre fédéral de la Santé Ding Wall.

Faute d'espace, d'abord, compte tenu ensuite que le projet de loi et de réglementation n'était pas encore déposé au moment de la rédaction de cet article et compte tenu, enfin, de l'incertitude qui prévaut quant aux suites qui seront données à ce dossier au plan fédéral et provincial, nous nous limiterons ici à souligner l'incohérence de ce moratoire qui, jusqu'à nouvel ordre, continue de laisser au seul libre-arbitre indi-

21 En janvier 1996, était créé un « comité consultatif sur le moratoire provisoire visant les techniques de reproduction alors qu'un groupe de travail sur les inducteurs de l'ovulation et un autre sur les embryons avaient été mis sur pied, ce qui pourrait laisser croire que Santé Canada sort enfin de l'attentisme. Cependant, sans contester la qualité et la compétence des personnes impliquées ni l'éventualité d'heureuses initiatives de leur part, il est difficile de ne pas souligner l'aberration d'un tel mandat, qui consiste, dans le cas du comité sur le moratoire » à " surveiller le respect d'un moratoire " volontaire », et à suivre l'apparition de nouvelles techniques (un peu comme on regarde passer le train), puisqu'il s'agit de conseiller la sous-ministre de la Santé sur l'opportunité d'inclure d'autres pratiques fortement discutables... dans ce même " moratoire ». Non seulement cela redouble la mystification, mais cela interroge profondément l'absence d'imputabilité et de transparence de telles instances ainsi que l'absence d'accès du publie à l'information. 
viduel et aux lois du marché des champs de recherche et d'intervention (modifications géniques germinales; hybrides; production d'embryons à partir d'ovules de fœtus et de cadavres ; ectogénèse, clonage d'embryons humains), si lourds de sens et de conséquences, qu'ils sont interdits dans nombre de pays; et au point de tolérer des pratiques comme le choix du sexe, l'industrie des "mères porteuses » et le commerce ou le troc d'ovules, de sperme et d'embryons ${ }^{22}$.

Après dix ans de débats publics, une controversée Commission royale de quatre ans et de 29,5 millions de dollars (Eichler 1993), prolongée par trois ans d'analyse de son rapport par une armée d' « experts » et de fonctionnaires, il était assez étonnant qu'un gouvernement confie à la seule conscience d'individus à la fois juges et parties des champs de recherche et d'intervention aussi explosifs, surtout dans un secteur où nombre de ces technologies, notamment la FIV et ses dérivés, ont été imposées par coups de force successifs, sans réflexion de fond quant à leur nature, leur bien-fondé (Trépanier, 1993 ; Vandelac, 1990a), leur pertinence et leurs effets en termes de santé publique ; sans évaluation scientifique (Klein, 1989 ; Wagner et Ste-Clair, 1989 ; OMS, 1990), sociale et éthique rigoureuse, le tout dans un étonnant tourbillon de sensationnalisme médiatique et de sidération publique abusivement interprétés comme de l'acquiescement (Vandelac, 1994d, 1995). En outre, dans un secteur où, notamment pour l'insémination artificielle (CRNTR, 1993, p. 507), même les lignes directrices des organismes professionnels (dont Erwin Chargaff, 1987, disait qu'elles étaient plus permissives que des manuels de broutage écrits par des chèvres) n'ont pas été respectées, n'était-il pas inquiétant de se contenter d'inviter simplement les promoteurs à s'abstenir de certaines pratiques, ce qui leur en laissait non seulement la liberté, mais également la possibilité de modeler davantage encore par associations professionnelles et lobbies interposés, les perspectives d'intervention gouvernementale ?...

22 Seuls les États-Unis sont aussi laxistes dans nombre de domaines, bien que le gouvernement américain se soit dispensé de laisser aussi explicitement carte blanche aux principaux intervenants. Pour certaines pratiques, l'Angleterre et certains États australiens sont également fort permissifs. 
Certes, la diffusion de ces technologies au Canada est moins débridée et moins caricaturale qu'aux États-Unis (Trip, 1996; Cowley, 1995) et moins pernicieuse qu'en Angleterre, où les prétendues instances d'encadrement (Bolton et coll., 1992) autorisent tout ou presque. En outre, la fécondation artificielle y est moins exponentielle qu'en France où l'on pratique dix fois plus de FIV alors que la population n'est que le double (Lancaster 1991) ${ }^{23}$.

Cependant, bien qu'au Canada il n'y ait pas encore, à notre connaissance, ni interventions géniques germinales, ni production d'embryons à partir de cadavres ou d'embryons, néanmoins les recherches sur l'ectogénèse animale ont déjà été amorcées ; les hybrides prennent forme d'animaux transgéniques (et non d'un nouveau bestiaire mythologique); la première communication sur le clonage d'embryons humains, réalisée grâce à une technique développée à l'Hôpital universitaire de London en Ontario et présentée par une équipe américaine à Montréal, en 1993, a d'abord reçu un prix du Congrès (Vandelac, 1994c) ; des entreprises privées de sexage ont ouvert leurs portes à Toronto, certaines y font le commerce et le troc d'ovocytes au Canada ou les écoulent aux États-Unis, une autre négocie des contrats de gestation ; la vente de sperme (qualifiée de compensation) est pratique courante et on peut acheter un embryon pour transfert dans l'utérus pour vingt mille dollars auprès d'une clinique américaine (MC, 1995).

Dans ce contexte, l'échec d'un tel moratoire « volontaire » tenait de l'évidence. D'ailleurs, dès son annonce, plusieurs promoteurs avaient riposté qu'ils l'enfreindraient, la SOGC et la SCFA en contestaient des aspects majeurs, alors que la seule mesure " contraignante », à savoir les coupures de fonds fédéraux aux contrevenants, était sans effets sur des pratiques déjà largement privées en plus d'inciter à la privatisation, compliquant d'autant tout contrôle.

Ainsi, «le meilleur pays du monde » qui se targue d'adopter des normes nationales de protection de la santé (Regush, 1993) a été l'un des derniers des pays développés à proposer d'interdire ou d'encadrer

23 Seul État, avec Israël à en assumer l'entièreté des coûts, ce qui n'est nullement indifférent à la prolifération de ces pratiques. 
des pratiques comme la modification génique germinale, l'ectogénèse, les hybrides et le clonage, déjà depuis longtemps condamnées ailleurs. Quant aux provinces, de qui relève l'essentiel de ces questions, elles ont « patienté dans l'azur » depuis dix ans ${ }^{24}$, tout en appuyant ce moratoire « volontaire » qui a permis d'élargir, de consolider et de privatiser cette industrie fulgurante du vivant, d'ici à ce qu'une éventuelle loi impose ses balises 25 .

\section{La mystification comme politique...}

$\underline{\text { Retour à la table des matières }}$

L'un des principaux paradoxes de ce moratoire a été d'annoncer, sous couvert d'une invitation à s'abstenir de certaines pratiques, les intentions véritables du législateur. À titre d'exemple, l'inclusion des « contrats ou accords à titre onéreux de maternité de substitution" dans ce moratoire visait en fait à donner un aval explicite aux contrats d'engendrement et de gestation non rémunérés, naïvement qualifiés d' « altruistes » et déjà en cours dans un hôpital de Calgary.

De la même façon, ce moratoire a avalisé le marché de la fécondation in vitro et la production d'embryons pour la recherche. Ainsi, " c'est pour s'assurer que les ovules utilisés pour la recherche effectuée au Canada sont ceux prélevés chez les femmes vivantes ayant donné leur consentement en tout état de cause » (MC: 9) [...] que l'exministre de la Santé " a demandé au corps médical et aux chercheurs du Canada de s'abstenir de recourir au prélèvement d'ovules sur des foetus ou des cadavres à des fins de don, de fécondation ou de recher-

24 Certaines comme l'Ontario s'étant retirées du financement intégral de la fécondation artificielle, alors que d'autres, comme le Québec, disposent de certaines dispositions législatives, notamment sur des questions de filiation...

25 Déposé peu avant la correction des épreuves de cet article, ce projet de loi marque certes de nets progrès par rapport au moratoire volontaire, mais il continue de porter certaines des zones d'ombres et d'ambiguïtés de ce moratoire. 
che » $(M C: 1)$. Cela signifierait-il qu'Ottawa, avec l'aval des provinces, autoriserait la création délibérée d'embryons humains pour la recherche 26 à partir de dons d'ovocytes ou suite à un prélèvement d'ovaires de femmes " consentantes ", d'ici à ce que, semble suggérer le texte accompagnant le moratoire, des mécanismes ne soient prévus pour qu'elles « consentent » avant leur décès ou pour le fœtus qu'elles auront produit... (MC: 9) ? Les ambiguïtés et contradictions de l'actuel projet de loi ne permettent aucunement de dissiper tous les doutes à ce sujet. Derrière l'interdit manifeste, et au nom sans doute des « valeurs morales et éthiques des Canadiennes et des Canadiens, et de la protection des groupes les plus touchés » (MC: 1), les pouvoirs publics avalise raient-ils ainsi en douce la proposition 188 du rapport Baird, suivant ainsi les traces des Britanniques qui, pour ce faire, utilisent déjà des ovules de fœtus et de cadavres 27 ), avec l'accord de l'organisme responsable de la réglementation. La production d'embryons comme modèles expérimentaux, matériel pour les lignées cellulaires et production de produits pharmaceutiques, constitue un enjeu économique clé, si bien que l'approvisionnement assuré en " matière première ", par le biais, notamment des prélèvements d'ovaires et des techniques de maturation des ovocytes, secondarise l'intérêt économique du clonage d'embryons, qu'il est plus facile alors d'interdire.

Dans le même ordre d'idées, ce moratoire reste muet sur les problèmes de circulation nord-américaine des gamètes et des embryons et face à l'économie américaine de la reproduction qui avec ses 300 cliniques de fertilité fonctionne virtuellement comme un libre marché de plus de 350 millions de dollars par année, au point de constituer dé-

26 Pour légitimer cette pratique, le rapport Baird parle de « zygote », tout comme en Angleterre 37 biologistes créaient de toutes pièces, à la fin des années quatre-vingt, l'expression fort contestée de "pré-embryon », visant également à tenter de légitimer ces recherches sur les embryons de moins de 14 ou 17 jours.

27 Certains milieux de recherche poussent parfois la perversité jusqu'à utiliser des sondages auprès d'étudiants universitaires de première année (18-21 ans) pour justifier « l'approbation du public (sic) dans l'utilisation d'ovaires d'embryons pour la recherche ou pour une intervention (sic) pour aider les couples infertiles ", alors que d'autres le font auprès de femmes en attente d'un don d'ovocytes (EDWARDS et BENHAM, 1994, p. 824 ; HOLT et coll., 1994 ; LIEBERMAN, 1994). 
sormais une branche de la médecine largement exempte de toute réglementation gouvernementale et des contrôles de coûts exercés par les compagnies d'assurances ». (TPN) (Trip, 1996). Le moratoire a également complètement ignoré la pratique charnière et carrefour de la fécondation in vitro, dont le rapport Baird soulignait pourtant que : "La façon dont la FIV est pratiquée à 1 heure actuelle est inacceptable. Il est, en effet, contraire à l'éthique et dangereux de permettre le recours à la FIV pour des indications où son efficacité n'a pas été démontrée » (ce qui est le cas de plus de la moitié des indications). Cela étonne d'autant plus que les coûts, les dérives, les impacts sociaux et les effets iatrogènes de la fécondation artificielle sont loin d'être négligeables. Ainsi, il n'est pas inutile de rappeler que les inducteurs d'ovulation, souvent utilisés tout azimut en reproduction artificielle, présentent un risque trois fois plus élevé de cancer ovarien pour les femmes ainsi traitées, alors que celles qui n'ont jamais été enceintes ont un risque 27 fois plus élevé (Whittemore, 1992). Une étude rétrospective menée à Seattle auprès de 3837 femmes investiguées pour « infertilité » (1974-1985) confirmait également que le risque de cancer ovarien chez les femmes traitées avec du citrate de clomiphène (Clomid) pendant 12 cycles et plus était de 11,1 comparativement à 2,3 chez les femmes "infertiles » non traitées. Cela n'est pas négligeable quand, selon le Food and Drug Administration, au moins 12,5 millions de ces produits auraient été prescrits aux États-Unis depuis le début des années soixante.

Bien qu'on puisse se réjouir que le Canada sorte enfin de son inqualifiable laxisme, soulignons néanmoins que si ce moratoire est utilisé comme marchepied, on risque fort de nouvelles entorses et une démarche boiteuse... Surtout si, comme le propose l'actuel projet de loi, les pratiques comme la fécondation in vitro, l'insémination par donneur, l'utilisation de tissu foetal, la conservation et le don d'ovules, de sperme et d'embryons humains ainsi que la recherche sur les embryons, notamment le diagnostic préimplantatoire font l'objet d'un cadre réglementaire dont les mécanismes d'élaboration et de surveillance sont pour l'instant aussi peu clairs que les questions d'imputabilité, de transparence et d'accès du public à l'information de la structure réglementaire supposée les encadrer. 
Dans ce contexte, nous ne pouvons que presser les pouvoirs publics d'approfondir l'analyse de ces questions tout en réitérant l'importance d'intervenir sur les sources mêmes de l'expansion des NTR (oestrogènes synthétisés, notamment); de clarifier la question du libreéchange et plus largement du commerce international en matière de production du vivant et de génétique, enfin de concevoir des outils de réflexion et d'encadrement pluridisciplinaires et interministériels plus novateurs, plus démocratiques et plus transparents que ne le sont certaines des instances environnementales pouvant servir d'inspiration.

Quant au Québec, soulignons que depuis les interventions du Conseil du statut de la Femme au milieu des années quatre-vingt (CSF, 1988) et depuis le rapport "tabletté » du MSSS en 1988, le gouvernement a été d'un total laxisme dans ce dossier, à l'exception de quelques nouvelles dispositions du Code civil qui ne sont pas toujours des plus heureuses (Deleury, 1995). Pourtant avec un des plus faibles taux de natalité et le plus haut de stérilisation volontaire des pays développés 28 ; avec un effritement sans précédent des solidarités familiales et sociales ; avec des problèmes manifestes de transmission intergénérationnelle (dont les hauts taux d'abandons scolaires et de suicide ne sont qu'un indice) ; avec une crise annoncée de la fertilité humaine et des effets particulièrement préoccupants au Québec, vu le lourd dossier des contaminants des grands lacs et du Saint-Laurent ; et enfin avec des problèmes aiguës des finances publiques, notamment en matière de santé ; qu'attend-on pour établir enfin une politique québécoise cohérente et intégrée en matière de santé reproductive ${ }^{29}$ ?

Sans ignorer le contexte canadien et américain, ces questions relèvent largement des juridictions provinciales, si bien qu'une telle poli-

28 Le Québec détient le plus haut taux de stérilisation volontaire du monde occidental (GUIBERT-LANTOINE, 1990), soit plus d'un million de personnes depuis 1971, à raison de 28000 à 40000 par année depuis 1976, (DUCHESNE, 1995, BENOÎT, 1996).

29 Une telle politique globale et intégrée de santé publique, dont la crédibilité repose sur des mécanismes démocratiques et transparents de débats publics, devrait tenir compte à la fois des enjeux éthiques, sociaux, économiques et démographiques que soulèvent les secteurs de la santé, de l'environnement, des sciences et technologies, de l'agriculture, de la justice, du travail, de l'éducation, de la famille et de la condition féminine. 
tique globale en santé reproductive ne peut y être inféodée. Par ailleurs, on ne peut utiliser l'alibi du caractère nécessairement global d'une telle politique pour retarder davantage l'application des aspects déjà plus étoffés en matière de périnatalité ou de planning familial, sous prétexte que les dossiers génétique et technologies de reproduction méritent d'être peaufinés, ce qui conduirait à une politique du fait accompli.

Il est en effet pour le moins paradoxal qu'un gouvernement aussi sensible aux questions d'identité nationale, notamment aux problèmes de langue et de culture qui en sont constitutifs, ignore toujours autant les questions d'identité personnelle et collective liées aux problèmes de santé reproductive. Sans prétendre que l'accessoire masque l'essentiel, les menaces à l'identité nationale ne méritent-elles pas d'être mises en perspectives quand c'est l'ordre des générations, l'espèce humaine et la conception des êtres et de l'humanité qui sont en question?

$\mathrm{Au}$ moment où la réification, l'instrumentalisation des êtres et l'éclatement des repères essentiels menacent la constitution psychique et l'identité des individus, comment peut-on continuer d'être aussi benoîtement à la remorque de la mise en marché du matériel et des fonctions reproductives, voire de la création délibérée d'embryons humains comme rats de laboratoire, bref, de se laisser ainsi piéger par l'emprise de technologies qui nous propulsent littéralement corps et âme hors de nous-mêmes... Si un éventuel projet de société implique de pouvoir se projeter dans l'avenir, au propre comme au figuré, en tant qu'individus et société, un tel projet implique d'abord le courage de la lucidité et une éthique partagée de la responsabilité...

\section{Louise VANDELAC}

Département de sociologie

Université du Québec à Montréal

C.P. 8888, Succ. Centre-ville

Montréal (Québec), Canada H3C 3P8 


\section{RÉSUMÉ}

$\underline{\text { Retour à la table des matières }}$

Alors que s'annonce une crise majeure de la fertilité humaine due notamment aux oestrogènes synthétisés, crise risquant d'accélérer la fuite en avant de technologies de la reproduction coûteuses, inefficaces, aux effets iatrogènes ci aux dérives problématiques qui bouleversent la conception des êtres humains et de l'humanité et qui fissurent les frontières mêmes de l'espèce, le Canada n'a toujours pour seule politique, après dix ans de débats et une Commission royale de trente millions de dollars, qu'un moratoire "volontaire ». Il demeure ainsi, jusqu'à nouvel ordre, l'un des rares pays à laisser explicitement au seul libre-arbitre individuel et aux lois du marché des champs de recherche et d'intervention comme la modification génique germinale ; les hybrides animal-humain; la production d'embryons à partir d'ovules de fœtus et de cadavres; l'ectogénèse, le clonage, le sexage, etc., pratiques si lourdes de sens que nombre de pays les ont interdites. Par son retard endémique à intervenir, « le meilleur pays du monde » serait-il en train de nous faire glisser dans « le meilleur des mondes »?

\section{SUMMARY}

In spite of the development of a major crisis in human fertility associated particularly with synthesized oestrogens, a crisis which risks accelerating the relentless pursuit of costly and inefficient reproduction technologies whose iatrogenic effects and problematical shifts in direction are having a profound effect on the conception of human beings and of humanity. and are threatening what we understand as the very frontier of the species, Canada's only policy so far, after ten years of debates and a $\$ 30$ million Royal Commission, is a "voluntary" moratorium. It is one of a few rare countries which has left fields of research such as genetic modification of the reproductive cell, animal-human hybrids, production of embryos from ovaries of foetuses and corpses; ectogenesis, cloning, gender determination, etc., all practices of such gravity that many countries have outlawed them, explici- 
tly and completely to individual free choice and the laws of the market. By its endemic lateness in reacting, would "the best country in the world" be letting us slip into the "best of all worlds"?

\section{RESUMEN}

Mientras se perfila una crisis mayor de la fertilidad humana asociada en particular a los eoestrógenos sintéticos, crisis que puede acelerar la fuga hacia adelante de las tecnologías de reproducción costosas, ineficaces, con efectos iatrógenos y desviaciones problemáticas, que altera la concepción del ser humano y de la humanidad y que desdibujan la frontera misma de la especie, Canadá tiene hasta ahova como única política, luego de 10 años de debates y de una Comision Real de \$30 millones, una moratoria « voluntaria ». De esta mariera, Canadá es uno de los pocos países que deja explicitamente al libre albedrío individual y a las leyes del rnercado, cíertos campos de investigación y de intervención como la modificación genética germinal ; los híbridos entre animales y humanos; la producción de embriones a partir de óvulos de fetos y cadáveres; la ectogenética, el « clonaje », el « sexaje », etc., prácticas tan cargadas de sentido que fueron prohibidas en numerosos países. ¿Por su retraso endémico para intervenir, « el mejor país del mundo » no nos estará Ilevando hacia el « mejor de los mundos »?

\section{BIBLIOGRAPHIE}

Retour à la table des matières

ATHEA, Nicole (1990), "La stérilité : une entité mal définie », Le Magasin des enfants, Collectif dirigé par Jacques Testart, Paris, Éd. François Bourin, pp. 37-71.

AUGER, J., KUNSTMANN, J.-M. CZYGLIK, F. et P. JOUANNET, « Decline in Semen Quality Among Fertile Men in Paris During 
the Past 20 years ", New England Journal of Medicine, 2 février, pp. 281-285.

\section{TECHNOLOGIES DE LA REPRODUCTION 73}

AUROUX, Maurice (1995), «Des handicaps créés par l'homme », La Recherche, n² 282, décembre, pp. 50-59,

BASEN, Gwynne (1992), On the Eighth Day, Perfecting Human Nature, 2 documentaires d'une heure sur les technologies de reproduction et la génétique, Office national du film, Montréal.

BASEN, Gwynne, EICHLER, Margrit et LIPPMAN Abby (dir.) (1993), Misconception: the Social Construction of Choice in the New Reproductive and Genetic Technologies, Hull, Voyageur Press.

BAUDRILLARD, Jean (1995), Le Crime parfait, Paris, Éd. Galilée, 204 p.

BENOIT, Monique (1996), Les Discours entourant le recours à la stérilisation chirurgicale au Québec. Une analyse de l'idéologie contraceptive et des enjeux de la ligature tubaire, projet de thèse, Département de Sociologie, UQAM, 39 p.

BERKOWITZ, Richard L. (1994), «The Mt. Sinai Experience with Multifetal Pregnancy Reduction », International Journal of Gynecology and Obstetrics, Supp. 3 to vol. 46, XIV FIGO World Congress, p. 45.

BOUCHARD Louise (1990), La Procréation artificielle, un futur à l'œuvre, mémoire de maîtrise en sociologie, Département de sociologie, Université de Montréal.

BUEKERT, Dennis (1996), « Laws Needed to Curb Rent-a-Womb Deals : Marleau », The Gazette, Montréal, 17 janvier. 
C.S.F. (1988), Sortir la maternité du laboratoire, Actes du forum international sur les nouvelles technologies de reproduction, Montréal, 29-31 octobre 1987, Concordia, Conseil du statut de la femme, Gouvernement du Québec, 423 p.

CASTLEMAN, Michael (1982), « Why Johnny Can't Have Kids », Mother Jones, avril.

CASTLEMAN, Michael (1996), « Down for the Count » Mother Jones, février, pp. 20-21.

CFAS (Canadian Fertility and Andrology Society) (1994), « Response to the Report of the Royal Commission on New Reproductive Technologies: A Summary », Can. Med. Assoc. J., vol. 151, n 10, pp. 1425-1432.

CFAS (Canadian Fertility and Andrology Society) (1995), « Response to the Voluntary Moratorium », Press Release, août.

CHARGAFF, Erwin (1987), «Engineering a Molecular Nigtmare », Nature, vol. 327, $\mathrm{n}^{\circ} 21$.

CLÉMENT, Dominique (1992), « Mamies-éprouvette », Le Point, $n^{\circ} 1029,6$ juin, p. 49.

CLIQUET, R.-L., BOSMAN, E, et VAN-DONGEN, W. (1989), Decreasing Fertility and Changing Relational Behavior in the OECD Member.

COREA, Genoveffa (1993), «Introduction », Misconceptions, Basen et coll. op. cit. vol. 1, pp. 13-26.

COWLEY, Geoffrey (1995), « The Future of Birth », News Week, 4 septembre.

CRNTR (1993), Un virage à prendre en douceur, vol. 1 et 2, Rapport final de la Commission royale d'enquête sur les nouvelles techniques de reproduction, $740 \mathrm{p}$. 
DECORNOY, Jacques (1992), « De l'irresponsabilité mortelle à la vraie maîtrise de la vie ", Le Monde Diplomatique, Manière de Voir, $\mathrm{n}^{\circ}$ 15, dossier L'Homme en danger de science, Paris, pp. 24-27.

DELAISI DE PARSEVAL, Geneviève et VERDIER Pierre (1994), Enfant de personne, Paris, Éd. Odile Jacob, 364 p.

DELEURY, Édith (1995), « Du corps que l'on est au corps que l'on a », Journal international de bioéthique, vol. 6, n 3, pp. 230-235.

De VILAINE, A.-M., GAVARINI, L. et Le COADIC, M., (dit.) (1986), Maternité en mouvement. Les Femmes la reproduction et les hommes de science, Grenoble, PUG et Montréal, Éditions Albert Saint-Martin, 244 p.

De VILAINE, Anne-Marie (1990), « Sortir de l'histoire », Le Magasin des enfants, Paris, François Bourin, pp. 194-216.

DREYFUS, Hubert et Paul RABINOW (1984), Michel Foucault: un parcours philosophique, Paris, Éditions Gallimard, 366 p.

DUCHESNE, Louis (1995), La Situation démographique au Québec, Québec, Statistiques démographiques, Publications du Québec, $200 \mathrm{p}$.

EDGAR, Harold et TURSZ, Thomas (1995), « Report on Human Gene Thérapy », Actes, 1995, Paris, Comité international de bioéthioque de l'UNESCO, vol. 1, pp. 29-51.

EDWARDS, Juliette H. et BENHAM, Jonathan (1994), «Views on Donated Ovarian Tissue », The Lancet, vol. 344, n 1, p. 824.

EICHLER, Margrit (1993), « Frankenstein meets Kafka: The Royal Commission on New Reproductive Technologies », Misconception, Basen, Eichler, Lippman, Op. cit., pp. 196-222,

FIVNAT (1993), Dossier Fivnat, Analyse des résultats 1989-1993, Données statistiques françaises de fécondation artificielle, 59 p. 
FOUCAULT, Michel (1983), Naissance de la clinique, Paris, Gallien/PUF (édition originale, 1963), 214 p.

GAUTHIER, Ursula (1990), « Voyage chez les sorciers de la vie », Le Nouvel Observateur. Va-t-on modifier l'espèce humaine?, Document $n^{\circ} 10$, Paris.

GAVARINI, Laurence (1987), Les Procréations artificielles aux regards de l'institution scientifique et de la Cité: la bioéthique en débat, thèse de doctorat, Université de Paris VIII.

GOUVERNEMENT DU CANADA (1991), Les Produits chimiques toxiques dans les Grands Lacs et leurs effets connexes, résumé, Environnement Canada, Ministère des Pêches et Océans, Santé et Bien-être social Canada.

GUIBERT-LANTOINE, Catherine (1990), « Révolutions contraceptives au Canada » Population, n² 2, pp. 361-398.

GUILLAUMIN, Colette (1978), " Pratique du pouvoir et idée de Nature, 1 et 2 », Questions féministes, n² 2, et 3, Paris.

HANMER, Jalna et ALLEN, Pat (1979), « La science de la reproduction: solution finale ? ", Questions féministes, $\mathrm{n}^{\circ} 5$.

HERMITTE, Marie-Angèle (1990), "L'embryon aléatoire », Le Magasin des enfants, Paris, Éditions François Bourin, pp. 327-367.

HERMITTE, Marie-Angèle (1992), «F.I.V. et tri des embryons », Génétique et Liberté, Paris, janvier-février, pp. 4-5.

HOLT, Elizabet, MADDISON, J. et SIDEBOTHAM, Mary (1994), "Views on Donated Ovarian Tissue », The Lancet, vol. 344, $\mathrm{n}^{\circ} 23$, pp. 824-825.

HUBBARD, Ruth et WALD, Elijah (1993), Exploding the Gene Myth, Boston, Beacon Press. 
IRIGARAY, Luce (1984), Éthique de la différence sexuelle, Paris, Éd. de Minuit, 199 p.

IRIGARAY, Luce (1987), Sexes et parentés, Paris, Éd. de Minuit, $222 \mathrm{p}$.

JEGOU, B., SPIRA, A. et JOUANNET, P. (1995), « La fertilité humaine est-elle menacée ? ", Contraception Fertilité Sexualité, n 9, pp. 513-515,

JONAS, Hans (1990), Le Principe responsabilité. Une éthique pour la civilisation technologique, Paris, Éd. du Cerf, 336 p.

KLEIN, Renate et ROWLAND, Robyn (1988), « Women as TestSites for Fertility Drugs : Clomiphene Citrate and Hormonal Cocktails », Reproductive and Genetic Engineering, Journal of Intemationa Feminist Analysis, Pergamon Press, vol. 1, no 3, pp. 251-274.

KLEIN, Renate D. (dir.) (1989), Infertility, Women Speak Out About Their Experience on Reproductive Medecine, Pandora Press, $328 \mathrm{p}$.

KOLATA, Gina (1996), « Study Finds a Way of Freezing and Reproducing Sperm Cells, Grown in Other Species », New York Times, 30 mai.

LABORIE, Françoise (1992a), Nouvelles Technologies de la reproduction (NTR) : risques pour la santé des femmes, AIDELF, Association internationale des démographes de langue française, Les Modes de régulation de la reproduction humaine, Colloque international de Delphes, Paris, PUF, 1994, 777 p.

LABORIE, Françoise (1992b), Nouvelles Technologies de la reproduction (NTR) : risques pour la santé des enfants, AIDELF, Les Modes de régulation de la reproduction humaine, op. cit.

LABRUSSE-RIOU, Catherine (1994), « Sciences de la vie et légitimité », Mélanges à la mémoire de Danièle Huet-Weller, Paris. 
LACHMANN, Peter (1995), " Some Considerations on Gene Therapy ", Actes, vol. 2, Comité international de bioéthique de l'UNESCO, Paris, pp. 53-57.

LANCASTER, Paul A. L. (1991), "Assisted Conception. Health Services and Evaluation ", International Journal of Technology Assessment in Health Care, vol. 7, n 4, pp. 485-499.

LÉRIDON, Henri (1992) «La stérilité : concepts et mesure. Le point de vue du démographe ", Contraception, Fertilité, Sexualité, vol. 20, n² 2, pp. 159-163.

LIEBERMAN, B. A. (1994), "Views on Donated Ovarian Tissue », The Lancet, vol. 344, n² 2, p. 824.

LIMOGES, Camille (1988), « Analyse évaluative et évaluation sociale des technologies : une pragmatique sociale ", Recueil des activités CIEST, UQAM, Département de philosophie, pp. 259-279.

LIPPMAN, Abby et VANDELAC, Louise (1992), « Cartographie et séquençage du génome humain, questions d'éthique et d'évaluation sociale des technologies ", Le Génome humain, une responsabilité scientifique asociale, Colloque Cartographie et séquençage du génome humain : la responsabilité scientifique et sociale, ACFAS 1991, Les Presses de l’Univesité Laval.

M.C. (1995) Morotoire canadien, communiqué de presse du 27 juillet 1995, suivi de la déclaration de la Ministre, d'une description des neuf techniques et pratiques visées par le moratoire, d'un glossaire et d'une liste des intervenants, Gouvernement du Canada, Santé Canada, $11 \mathrm{p}$.

M.F.P.F. (1989) (Mouvement français pour le Planning familial), L'Ovaire-Dose, Les Nouvelles Méthodes de procréation, Paris, Éd. Syros, Alternative.

M.S.S.S. (1988), Rapport du comité de travail sur les nouvelles technologies de reproduction humaine, Ministère de la Santé et des 
Services sociaux, Direction générale de la planification et de l'évaluation, Gouvernement du Québec, 120 p.

M.S.S.S. (1993), Politique de périnatalité, Ministère de la Santé et des Services sociaux, Gouvernement du Québec, 101 p.

MARCUS-STEIFF, Joachim (1994), La Mesure des taux de « succès » de la FIV (fécondation in vitro et transfert d'embryon), AIDELF, Les Modes de régulation de la reproduction humaine, Paris, PUF, pp. 637-646.

MeINNIES-RAE, Rick (1995), "Commerce in Human Eggs », Transcription d'une émission radio de la CBC, The World at Six, 1er novembre, réf. : 74008.18.

MESSING, Karen et OUELLETTE, Gail (1991), Infertilité et milieu de travail, CINBIOSE, Centre pour l'étude des interactions biologiques entre la santé et l'environnement, Institut de recherche en santé et en sécurité du travail, Montréal, 15 novembre, 104 p.

MORIN, Edgard (1990), Science avec conscience, Paris, Fayard, coll. «Points/Sciences ».

O'BRIEN, Mary (1987), La Dialectique de la reproduction, traduction de The Politics of Reproduction (Boston, Routledge and Kegan Paul, 1981), Montréal, Éd. du Remue-Ménage.

ORENSTEIN, Peggy (1995), « Looking for a Donor to Call Dad », New York Times Magazine, 19 juin.

OTA (1987), Office of Technology Assessment, Artificial Insemination, Practice in the United States, Background Paper, Congress of United States, Library of Congress, Washington, $112 \mathrm{p}$.

OTA (1988), Office of Technology Assessment, Infertility, Medical and Social Choices, Congress of United States, Library of Congress, Washington, $402 \mathrm{p}$. 
RAMONET, Ignacio (1995), «Éditorial », Le Monde Diplomatique Manières de Voir, $\mathrm{n}^{\circ}$ 27, Paris.

REBICK, Jules (1993), «Is the issue Choice ?», Misconception, Basen, Eichler, Lippman (dir.), vol. 1.

REGUSH, Nicholas (1993), Safety Last. The Failure of the Consumer Health Protection System in Canada, Toronto, Key Porter Books, $212 \mathrm{p}$.

RELIER, J. P. (1992), « Résultats de la prise en charge de la stérilité. Le point de vue du pédiatre », Contraception, Fertilité, Sexualité, vol. 20, n 2, pp. 136-141.

ROBERTSON, John A. (1994), Children of Choice: Freedom and The New Reproductive Technologies, Princeton, N.J., Princeton University Press, $281 \mathrm{p}$.

ROCHON, Madeleine (1986), « Stérilité et infertilité: deux réalités », Santé Société, été, pp. 33-36.

ROSTAND, Jean (1966), Maternité et biologie, Paris, Éd. Gallimard, $179 \mathrm{p}$.

SEIBEL, Michelle M. (1994), « Cadaveric Ovary Donation », The New England Journal of Medicine, p. 796.

SEIDEL, G. F. Jr. (199 1), «Embryo Transfer : The Next 100 Years », 1991 Annual Conference International Embryo Transfert Society.

SOGC (1995), «The Society of Obstetricians and Gynaecologists of Canada Supports Marleau's Initiatives on Reproductive Technologies », communiqué de presse, 27 juillet.

SPIRTAS, R. et coll. (1993), «Fertility Drugs in Ovarian Cancer: Red Alert or Red Herrings ? » Fertility and Sterility, vol. 59, n 2 pp. 291-293. 
STE. CLAIR STEPHENSON, Patricia A. (1991), «The Risks Associated With Ovulation Induction », Iatrogenics, no 1, pp. 7-16.

TESTART, Jacques (1992), Le Désir du gène, Paris, Éditions François Bourin, 281 p.

TESTART, Jacques (dit.) (1990), Le Magasin des enfants, Paris, Éditions François Bourin, 338 p.

THIBON-CORNILLOT, Michel (1992), Les Corps transfigurés. Mécanisation du vivant et imaginaire de la biologie, Paris, Éditions du Seuil, $311 \mathrm{p}$.

TRÉPANIER, Isabelle (1993), La Fécondation in vitro et transfert d'embryons : traitement ou expérimentation? Le Cas de cinq rapports nationaux: Warnock, Benda, 5 sages, MSSS et Lenoir, mémoire de maîtrise, Département de sociologie, UQAM.

TRIP, Gabriel (1996), « The Fertility Market », New York Times, série de quatre articles du 7 au 10 janvier.

VACQUIN, Monette (1987), "L'amère victoire ", Autrement, no 90, La mère, pp. 148-158.

VACQUIN, Monette (1990a), «De la science au délire», Le Monde, Paris, 10 mai, p. 5.

VACQUIN, Monette (1990b), «Frankenstein et l'enfantement du contemporain », Libération, Paris, 9 mai, p. 5.

VANDELAC, Louise (1988a), L'Infertilité et la stérilité: l'alibi des technologies de procréation, thèse de sociologie, Doctorat Nouveau Régime, Université Paris VII, Jussieu, 504 p.

VANDELAC, Louise (1988b), «Technologies de procréation et «biologisation » de la paternité », Actes de la table ronde internationale de l'APRE (Atelier production-reproduction), Rapports sociaux de sexes : problématiques, méthodologies, champs d'analyse, CNRS, Paris 24-26 novembre 87, pp. 241-250. 
VANDELAC, Louise (1989a), "Stérilité iatrogène, stérilisation volontaire et infertilité idiopathique: l'élargissement sans fin des clientèles des technologies de procréation ", L'Ovaire-dose, MFPF, pp. 151-176.

VANDELAC, Louise (1989b) «La face cachée de la procréation artificielle », La Recherche, Paris, vol. 20, septembre, pp. 1112-1124.

VANDELAC, Louise (1990a), «Technologies de procréation: du redéploiement de la médecine à l'emprise du biopouvoir », Du privé au politique : la maternité et le travail comme enjeux des rapports de sexes. De l'expérience de la maternité à J'enceinte des technologies de procréation. Francine Descarries, Louise Vandelac, et G. Gagnon. (dir.), Montréal, GIERF/CRF, UQAM, octobre, pp. 243-260.

VANDELAC, Louise (1990b), «L'embryo-économie du vivant... ou du numéraire aux embryons surnuméraires » Le Magasin des enfants, pp. 117-139.

VANDELAC, Louise (1994a), "The Baird Commission, From " Acces » to Reproductive Technologies to the "Excesses » of Practitioners ; or, the Art of Diversion and Relentless Pursuit... ", in Misconception, vol. 1 pp. 253-272.

VANDELAC, Louise, (1994b), «Technologies de reproduction: techniques de régulation à la hausse ou véritables montagnes russes? ", Les Modes de régulation de la reproduction humaine, incidences sur la fécondité et la santé, Colloque de l'AIDELF, Paris, PUF, pp. 399-414.

VANDELAC, Louise, (1994c), « Clonage médiatique... Life is Xerox and I am just a copy », Interface, Dossier Clonage d'embryons humains, vol. 15, $n^{\circ} 1$, pp. 39-41.

VANDELAC, L., BARALDI, R. et FORTIN, E. (1994d), « La reproduction médiatiquement assistée ", L'Agora, vol. $1 \mathrm{n}^{\circ} 4$, pp. 1 et pp. 36-39. 
VANDELAC, Louise (1994e), « The Impact of Technology on Human Conception and the Concept of Humanity », Canada's Mental Health, vol. 42, n² 2, Health Canada, Ottawa, pp. 19-23.

VANDELAC, Louise et Francine DESCARRIES (I994f), «L'engendrement du savoir ", Cahiers de recherche sociologique, $\mathrm{n}^{\circ} 23$, Département de sociologie, UQAM, pp. 5-24.

VANDELAC, Louise (1995), « Pour une analyse critique des dispositifs d'évaluation scientifique, éthique et sociale des technologies de reproduction ", Ruptures, Revue transdisciplinaire en santé, GRIS, Faculté de Médecine, Université de Montréal, vol. 2, n 1, pp. 74-101.

VIGNAUX, Georges (1977), " Les machineries du vivant. Arguments et représentations ", Discours biologique et ordre social, Achard, Chauvenet, Lage, Lentin, Nève, Vignaux (dir.), Paris, Éd. du Seuil, 284 p.

W.H.O. (1990), World Health Organization, Regional Office for Europe, Consultation on the Place of In Vitro Fertilization in Infertily Care, Copenhague, Summary Report, 18-22 juin, 7 p.

WAGNER, Marsden G. et STE-CLAIR, Patricia A. (1989), The Lancet, 28 octobre, pp. 1027-1029.

WHITTEMORE, A. S, et coll. (1992), The Collaborative Ovarian Cancer Group, "Characteristics Relating to Ovarian Cancer Risk: Collaborative Analysis of Twelve US Case-Control Studies II. Invasive Epithelial Ovarian Cancer in White Women ", American Journal of Epidemiology, vol. 136, $\mathrm{n}^{\circ}$ 10, pp. 1194-1203

WRIGHT, Lawrence (1996), "Silent Sperm, A Reporter at Large » The NewYorker, 15 janvier, pp. 42-55.

Fin du texte 